Abstracts: Session I

tive gene expression intensities, ratios or both are measured in order to quantify the gene expression level relative to its reference sample. Our early reports discussed a systematic data extraction algorithm in which a unique method of extracting gene expression intensities and ratios along with an adaptive ratio confidence interval, measurement qualities of gene expression ratios and intensities were presented. In many methods of gene expression data analysis, only expression ratios or normalized intensities are employed because of insufficient assessment at the individual data points (clones). Common practice dictates that data derived with poor measurement quality-such as expression ratios derived from weak reference expression levels or noise-corrupted measurements—shall not be used in the analysis. We present an automatic decision-making process for various algorithms, such as gene expression clustering and classification, in which gene expression ratios and intensities are chosen to participate in the analysis according to their measurement quality, expression signal-to-noise ratio relative to the reference channel and other parameters derived from cDNA microarray image analysis software.

Chen, Zhong [36]

\section{Molecular profiling of metastatic tumor progression of a murine squamous cell carcinoma by differential display and CDNA microarray reveals dysregulated expression of genes related to the nuclear factor- $k b$ signal pathw ay}

\author{
Gang Dong ${ }^{1}$, Elena Loukinova ${ }^{1}$, Zhong Chen ${ }^{1}$, Lisa Gangi ${ }^{2}$ \\ Edison Liu² \& Carter Van Waes ${ }^{1}$ \\ ${ }^{1}$ National Institutes of Health, Bethesda, Maryland, USA National Cancer \\ Institute, National Institutes of Health, Bethesda, Maryland, USA
}

To identify changes in gene expression associated with tumor progression and metastasis in vivo, we investigated differential gene expression in a metastatic squamous carcinoma model established in syngeneic mice, including a tumorigenic line PAM 212, and metastatic sublines derived from PAM 212 tumors, using mRNA differential display (DD) and cDNA microarrays. Using DD, seventy-two candidate cDNAs were detected, and thirty-four cDNAs were confirmed to be differentially expressed by northern blotting analysis. Global mRNA expression profiles were generated using an NCI mouse Oncochip composed of four thousand elements representing known genes and ESTs, plus 57 of the candidate cDNAs detected by DD to facilitate data validation. Clustering analysis of array results from metastatic cell lines and tumors identified a subset of genes that exhibited increased expression in the metastases, revealed that 22 unique clones are highly homologous to previously identified genes, and nine novel cDNAs. Strikingly, $10 / 22$ of the genes identified have been associated with activation of the Nuclear Factor-kB signal transduction pathway. One of the genes identified, Gro-1, was recently confirmed to promote tumor growth, metastasis and angiogenesis of SCC in vivo in a separate report. These results demonstrate that early response pathway components and down stream genes related to NF- $\mathrm{KB}$ are expressed with metastatic tumor progression. Functional genomic approaches may promote a better understanding of the repertoire of related genes and molecular pathways involved in tumor progression and metastasis.
Chodosh, Lewis

[37]

\section{Functional analysis of mammary development using oligonucleotide microarrays}

Stephen Master, Jennifer Hartman, Alexander Stoddard, Elizabeth Keiper, Susan Moody, Celina D'Cruz $\&$ Lewis Chodosh

University of Pennsylvania School of Medicine, Philadelphia, Pennsylvania, USA

Epidemiological and animal studies clearly demonstrate that reproductive events such as puberty, pregnancy and parity play a critical role in the determination of breast cancer risk. We have carried out messenger RNA expression profiling using high-density synthetic oligonucleotide microarrays to identify genes that are differentially expressed between various developmental stages of the murine mammary gland. The use of DNA microarrays to study vertebrate development presents unique analytical challenges compared with expression profiling of homogeneous cell populations. These challenges include accounting for the impact of complex changes in the abundance of multiple cell types on gene expression profiles, as well as identifying functionally relevant patterns of gene expression in the absence of detailed prior knowledge either of the developmental system or of the genes expressed. In order to address these challenges, we have developed a general approach that permits the unbiased identification of biologically relevant patterns of gene expression by identifying statistically significant associations between clustered gene expression patterns and functional gene categories. We have tested the applicability of this approach by analyzing the expression of approximately 5,500 genes during 13 stages of murine mammary gland development. Our findings confirm the utility of this method by demonstrating the ready identification of cellular processes and pathways of known importance in mammary development, as well as shifts in the relative abundance of different cell types within the gland. This approach permitted the identification of genetic pathways with previously unsuspected patterns of developmental regulation, including those involved in fatty acid metabolism, angiogenesis and extracellular matrix synthesis. Our results demonstrate the ability of this analytical approach to suggest new hypotheses regarding mammary development and indicate that this approach will be broadly applicable to the study of complex tissues.

Chuang, Y. Eric

$[38]$

\section{Effects of p53 in human lymphoblast cells following ionizing radiation using $7 \mathrm{~K}$ cDNA microarrays}

Y. Eric Chuang ${ }^{1}$, Howard Liber ${ }^{2}$, Louis Staudt ${ }^{1}$

$\&$ James Mitchell ${ }^{1}$

${ }^{1}$ Division of Clinical Sciences, National Cancer Institute, National Institutes of Health, Bethesda, Maryland, USA

${ }^{2}$ Department of Radiation Oncology, Massachusetts General Hospital, Boston, Massachusetts, USA

Genomic instability is a characteristic of many human cancers. It has been well documented, utilizing endpoints such as karyotypic instability and gene amplification, that alterations in the tumor suppressor p53 are related to genomic instability at the chromosomal level. The p53 protein has been implicated in multiple cellular responses related to DNA damage, including apoptosis, cell cycle control and DNA replication, repair and transcription. Alterations in any of these processes could be related to increased genomic instability. We previously compared radiation-induced mutagenicity among human B lymphoblast cell lines with dif- 